\title{
Características Tecnológicas de la Fibra de Llama (Lama glama) Chaku antes y después de Descerdar
}

\author{
Technological Traits of the Chaku Llama Fibre Before and After Dehairing \\ Flor de María Laime Huarcaya ${ }^{1}$, Rubén Pinares Huamaní2, Valeriano Paucara \\ Ocsa² $^{2}$ Virgilio Machaca Machaca ${ }^{2}$, Edgar Carlos Quispe Peña ${ }^{3,4}$
}

\section{Resumen}

Se evaluaron cinco características tecnológicas de la fibra de llama: diámetro medio de fibra (MDF), coeficiente de variación de MDF (CVMDF), factor de confort (FC), índice de curvatura (IC) y finura al hilado (FH) antes y después de descerdar. Se tomaron muestras de $10 \mathrm{~g}$ de fibra de vellones de 227 llamas Chaku de la región Apurímac, Perú. Las fibras sin descerdar y descerdadas fueron analizadas con el equipo OFDA 2000 (Optical Fibre Diameter Analyser). Se consideraron las variables sexo y edad (1-2, $>2$ años) en el análisis estadístico a través de un diseño completamente al azar con arreglo factorial. Los resultados indican que la fibra descerdada es de mejor calidad, disminuyendo la $\mathrm{MDF}(0.70 \mu \mathrm{m})$, el CVMDF $(1.8 \%)$ y la FH $(1.06 \mu \mathrm{m})$ e incrementando el FC $(2.74 \%)$ y el IC $\left(4.66^{\circ} / \mathrm{mm}\right)$. Asimismo, el sexo no tuvo un efecto significativo en las características tecnológicas de la fibra pero las llamas juveniles presentaron mejor calidad de fibra. Se concluye que el descerdado y la edad de la llama tienen efectos significativos sobre la finura y su variación, el factor de confort, el índice de curvatura y la finura al hilado.

Palabras clave: calidad textil, fibra fina, fibra gruesa, camélido sudamericano

\section{AbSTRACT}

Five technological characteristics of llama fiber were evaluated: average fibre diameter (AFD), the coefficient of fibre diameter (CVD), the comfort factor (CF), the fibre curvature (FC) and the spinning fineness (SF) before and after dehairing. Fibre samples (10 g) were collected from fleece of 227 llamas Chaku from the Apurimac region of Peru. Fiber samples before and after dehairing were analyzed with the OFDA 2000

\footnotetext{
${ }^{1}$ Municipalidad Provincial de la Convención, La Convención, Cuzco, Perú

${ }^{2}$ Facultad de Medicina Veterinaria y Zootecnia, Universidad Nacional Micaela Bastidas de Apurímac, Abancay, Perú

${ }^{3}$ Universidad Nacional Autónoma de Chota, Cajamarca, Perú

${ }^{4}$ E-mail: edgarquispe62@yahoo.com
}

Recibido: 18 de mayo de 2015

Aceptado para publicación: 2 de octubre de 2015 
(Optical Fibre Diameter Analyser). Sex and age (1-2 years and > 2 years old) were considered as variables and the data was analyzed in a randomized complete design with a factorial arrangement. The results showed that dehaired llama fibre showed better quality, less $\operatorname{AFD}(0.70 \mu \mathrm{m}), \mathrm{CVD}(1.8 \%)$ and $\mathrm{SF}(1.06 \mu \mathrm{m})$ and better $\mathrm{CF}(2.74 \%)$ and $\mathrm{FC}\left(4.66^{\circ} \mathrm{mm}\right)$. Sex had no significant effect on the technological characteristics of the fibre but younger animals had better quality fibre. It is concluded that dehairing and age have significant effects on lama fibre in relation to fineness and its variation, comfort factor, fibre curvature and spinning fineness.

Key words: textile quality, fine fibre, coarse fibre, South American camelids

\section{INTRODUCCIÓN}

La llama (Lama glama) es el camélido doméstico más grande y se encuentra adaptada a un amplio rango de condiciones medioambientales, siendo de gran importancia para los ecosistemas de los Andes, por sus múltiples posibilidades de uso como productor de fibra y carne, su empleo en el transporte de carga y el múltiple uso del estiércol, así como por su importancia cultural (Stemmer et al., 2005; Quispe et al., 2009).

Perú es el segundo productor de llamas a nivel mundial, con 746269 animales distribuidos en más de 55000 unidades agropecuarias, donde prácticamente la mitad corresponde al tipo Chaku o lanuda y la otra mitad a Qara o pelada. La región de Puno tiene la mayor cantidad de llamas (32\%), mientras que la región de Apurímac ocupa el séptimo lugar a nivel nacional (4.8\%) (INEI, 2012).

El uso eficiente de la llama, no solo como productor de carne, sino también para fibra, donde se le podría dar un valor agregado que conlleve a la venta de fibra cardada (sliver), fibra peinada (top) y prendas, mejoraría sustancialmente los ingresos del productor llamero (Pinares et al., 2014; Quispe et al., 2015).

La fibra de la llama es gruesa y con alto contenido de pelo en el vellón (Cochi, 1999), de allí que sea muy poco aprovechada. La poca cantidad de fibra que se esquila es utili- zada mayormente para el consumo doméstico y solo un pequeño porcentaje es comercializado en los mercados locales (Quispe $e t$ al., 2015). Los compradores y productores de fibra coinciden en que existe una demanda de fibra de llama que no se aprovecha debido a bajos índices de extracción y por la fluctuación en la calidad y cantidad (Stemmer et al., 2005).

En forma general, se sabe que el vellón de la llama tiene dos tipos de fibra: las cerdas (fibra gruesa, fibra objetable o pelo) y el down (fibra fina), siendo esta última de buena calidad (Frank et al., 2011; Mamani et al., 2012). La fibra en broza puede tener valores de diámetro medio de fibra (DMF) menores a 23 $\mu \mathrm{m}$ (Coates y Ayerza, 2004), pero con tendencia a aumentar con la edad y la esquila (Sunari, 1986). En los casos donde el destino de la fibra es la confección de prendas, se aconseja el descerdado para facilitar el hilado y teñido (Rodríguez, 2007; Pilco et al., 2013), además de permitir obtener una mayor proporción de fibras finas (Quispe et al., 2009), mejorando así la calidad textil de la fibra (Rodríguez, 2007; Siguayro, 2009; Pilco et al., 2013; Pinares et al., 2014).

Se ha determinado que la finura de la fibra de llama argentina sería similar a la señalada para llamas peruanas (Quispe et al., 2009, Pinares et al., 2014) y bolivianas, pues presentan un diámetro medio de $23.27 \mu \mathrm{m}$, donde cerca del $78 \%$ es menor a $25 \mu \mathrm{m}$ (Martínez et al. 1997; Cochi, 1999; Rodriguez, 2007). Asimismo, Stemmer et al. (2005) señalan un diámetro medio de $22.16 \mu \mathrm{m}$. Otros 
estudios señalan, además, diámetros promedios similares a la fibra de la alpaca peruana. Así, Montes et al. (2008), en una muestra de 203 alpacas de ocho comunidades de Huancavelica indicaron que el $60 \%$ de los animales presentaron un diámetro $\leq 23 \mu \mathrm{m}$ y un diámetro promedio de $22.70 \mu \mathrm{m}$, en tanto que Adot et al. (2008) señalan que el 55\% de la fibra de alpaca peruana posee un diámetro inferior a $26 \mu \mathrm{m}$, y Quispe et al. (2009) mencionan que más del $70 \%$ de la fibra de alpaca huancavelicana se encuentra en la categoría extrafina.

Desde el punto de vista textil, el descerdado produce cambios estructurales en el vellón, ya que la extracción de las fibras más gruesas, más largas y más rectas (ofensivas) reduce la sensación de picazón y mejora la confortabilidad de las prendas, ajustándola a mejores estándares de calidad (Wang et al., 2003; Frank, 2011; Frank et $a l ., 2011)$, ya que se reduce el diámetro medio y coeficiente de variación de la fibra, así como la finura al hilado, incrementándose, a la vez, el factor de confort e índice de curvatura (Pinares et al., 2014).

A pesar de las investigaciones existentes que indican que el descerdado produce cambios estructurales y mejora la calidad textil de la fibra de la llama Chaku, aún no se dispone de información precisa sobre sus características por efecto del descerdado; de allí que el presente trabajo tuvo como objetivo evaluar sus características tecnológicas (diámetro medio de fibra, coeficiente de variación de MDF, factor de confort, índice de curvatura y finura al hilado) antes y después del descerdado de la fibra, así como evaluar el efecto de la edad y sexo sobre estas variables.

\section{Materiales y Métodos}

Se tomaron muestras de fibra $(10 \mathrm{~g})$ de 227 vellones de llamas Chaku, que no habían sido esquiladas en los años anteriores. Las llamas eran del estrato juvenil, de 1 a 2 años
( $\mathrm{n}=113)$ y adultas, mayores de 2 años $(\mathrm{n}=114)$, tanto hembras ( $\mathrm{n}=112)$ como machos $(\mathrm{n}=115)$, provenientes de la comunidad de Iscahuaca, distrito de Cotaruse, provincia de Aymaraes, región de Apurímac. La zona corresponde a puna seca y se encuentra a una altitud de 3700 a $5300 \mathrm{msnm}$. La esquila se hizo entre octubre a diciembre de 2012.

Las muestras de fibra se analizaron con el equipo OFDA 2000, en el Laboratorio de Fibras y Lanas de la Universidad Nacional de Huancavelica, a una temperatura de $20{ }^{\circ} \mathrm{C}$ y humedad relativa de $65 \%$. Se determinó el diámetro medio de fibra (MDF), coeficiente de variación de MDF (CVMDF), factor de confort (FC), índice de curvatura (IC) y finura al hilado (FH). El descerdado se realizó manualmente, de acuerdo al procedimiento descrito por Frank et al. (2012). Las medidas se realizaron antes y después del descerdado.

En el análisis de los datos, se realizó un análisis exploratorio para detectar 'outliers' pudiendo descartarse dos registros. Los datos correspondientes al FC se sometieron a una transformación trigonométrica (arcoseno), para posteriormente evaluarse la distribución normal y homogeneidad de varianza de los datos, mediante las pruebas no paramétricas de Anderson-Darling y Levene respectivamente (Martínez, 2009), mientras que la independencia de los errores fue evaluada mediante ploteo de los errores.

Las características tecnológicas fueron evaluadas con los paquetes estadísticos «Psych» y R (Revelle, 2015), mientras que el efecto de la fase (sin descerdar, descerdada), clase (grupo etario) y sexo sobre las características en estudio se hizo mediante un análisis de varianza con un modelo aditivo lineal correspondiente a un diseño completamente al azar con arreglo factorial de $2 \times 3$. Asimismo, se usó este mismo diseño para evaluar el efecto de la clase y el sexo para muestras de fibra sin descerdar y descerdada, pero con un arreglo factorial de $2 \times 2$. En todos los análisis se utilizó el paquete estadístico R v. 3.1.2. (R Core Team, 2014). 
Cuadro 1. Características tecnológicas ${ }^{1}$ de la fibra de llama Chaku antes y después de descerdar $(n=227)$

\begin{tabular}{lcccc}
\hline & Promedio & $\begin{array}{c}\text { Error } \\
\text { estándar }\end{array}$ & Mínimo & Máximo \\
\hline Fibra sin descerdar & 22.49 & 0.24 & 16.18 & 41.42 \\
MDF $(\mu \mathrm{m})$ & 22.14 & 0.19 & 14.70 & 31.00 \\
CVMDF $(\%)$ & 89.53 & 0.96 & 6.30 & 100.00 \\
FC $(\%)$ & 37.77 & 0.56 & 15.10 & 66.70 \\
IC $(\% / \mathrm{mm})$ & 22.17 & 0.25 & 16.07 & 41.65 \\
FH $(\mu \mathrm{m})$ & & & & \\
Fibra descerdada & 21.79 & 0.22 & 15.91 & 38.77 \\
MDF $(\mu \mathrm{m})$ & 20.34 & 0.14 & 14.80 & 26.20 \\
CVMDF $(\%)$ & 92.27 & 0.88 & 9.70 & 100.00 \\
FC $(\%)$ & 42.43 & 0.60 & 14.80 & 65.60 \\
IC $(\% \mathrm{~mm})$ & 21.11 & 0.22 & 15.47 & 38.07 \\
FH $(\mu \mathrm{m})$ & & & & \\
\hline
\end{tabular}

${ }^{1}$ MDF; diámetro medio de fibra; CVMDF: coeficiente de variación de MDF; FC: factor de confort; IC: índice de curvatura; FH: finura al hilado

\section{Resultados y Discusión}

\section{Características Tecnológicas de la Fibra}

Los valores promedio ( \pm error estándar) de la fibra sin descerdar y descerdada se muestran en el Cuadro 1. Asimismo, algunas fibras llegaron a tener una MDF de $16 \mu \mathrm{m}$ con una buena homogeneidad (CVMDF $<15 \%)$ luego del descerdado.

Los valores de MDF de las fibras sin descerdar resultaron similares a los reportados en otros estudios (Stemmer et al., 2005; Cancino et al., 2006; Rodríguez, 2007; Hick et al., 2009; Quispe et al., 2009; Quispe, 2014). No obstante, Martínez et al. (1997) y Cochi (1999) indican valores más altos de MDF (entre 31.2 y $36 \mu \mathrm{m}$ ), lo cual se debería al tipo, origen y edad de los animales en estudio. Sunari (1986) demostró en llamas que el diámetro de la fibra aumenta con la edad y el número de esquilas. Por otro lado, estos re- sultados podrían indicar que las llamas de la zona en estudio tienen buenas características respecto a la finura de la fibra.

En el caso de la fibra descerdada, los resultados fueron ligeramente superiores a los reportados por Siguayro (2009) y Quispe (2014) en llamas de primera esquila, lo cual resulta concordante pues animales jóvenes producen fibras con buena finura. No obstante, los valores obtenidos fueron inferiores al estudio de Frank et al. (2012), lo que respaldaría la información sobre el buen potencial que tienen las llamas de la región de Apurímac en la producción de fibra, dado que la fibra descerdada podría ser considerada de calidad textil similar o mejor a la fibra de alpaca (Siguayro, 2009; Mueller et al., 2010; Quispe, 2014).

$\mathrm{Al}$ analizar el Cuadro 1 y la Figura 1, se puede indicar que IC y el FC se incrementan en $4.66 \% \mathrm{~mm}$ y $2.74 \%$, respectivamente, mientras que el DMF, el CVDMF y la FH 

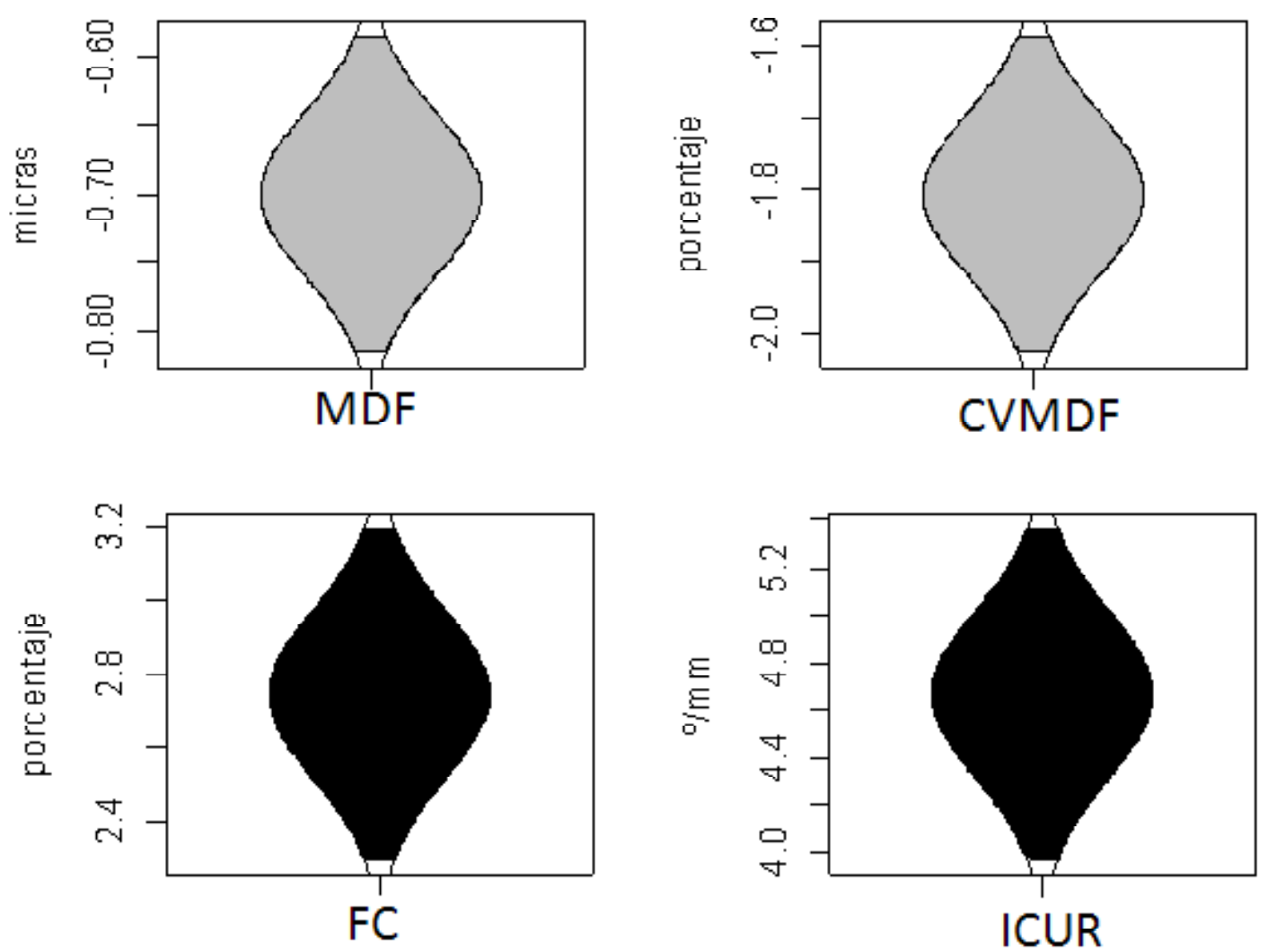

Figura 1. Intervalos de confianza (alfa=0.05) de los cambios de cuatro características tecnológicas de la fibra de llama Chaku por efecto del descerdado (MDF = Media de diámetro de fibra, CVMDF = Coeficiente de variación de la MDF, FC = Factor de confort, $\mathrm{IC}=$ Índice de curvatura)

disminuyen en $0.7 \mu \mathrm{m}, 1.8 \%$ y $1.06 \mu \mathrm{m}$, respectivamente, teniendo la mayor variación relativa el IC $(12.33 \%)$ y la menor el FC (3.06\%). Por otro lado, Pinares et al. (2014) y Quispe (2014), reportaron mayor variación relativa del factor de confort (23.0 y $8.6 \%$, respectivamente).

El CVMDF resultaría ser adecuado para las exigencias de la industria textil, siendo incluso inferior a valores de 23.48 a $28.10 \%$ reportado en alpacas (McGregor, 2002; Wang et al., 2003; McGregor y Butler, 2004; Lupton et al., 2006). Esta característica tiene alta influencia sobre algunas propiedades requeridas en la industria textil (Lupton et al., 2006), resultando conveniente un valor menor a $24 \%$, pues a partir de este valor, la finura al hilado disminuye $1 \mu \mathrm{m}$ por cada $5 \%$ de disminución.

\section{Efecto de la Fase, Clase, Sexo e Interac- ciones}

La fase de procesamiento tuvo un efecto significativo sobre todas las características tecnológicas en estudio, lo cual demuestra que el descerdado mejora la calidad de la fibra, tal y como ha sido reportado (Cochi, 1999; Frank, 2011; Pilco et al., 2013; Pinares et al., 2014; Quispe, 2014). El descerdado permitió obtener fibras más finas y homogéneas, con menor factor de picazón y mayor índice de curvatura, aspecto que debe ser 
Cuadro 2. Efecto de la fase (sin descerdar y descerdada), clase (juvenil y adulto), sexo (macho y hembra) y sus interacciones sobre las características tecnológicas de la fibra de llama Chaku

\begin{tabular}{lccccccccccc}
\hline & \multicolumn{4}{c}{ MDF } & \multicolumn{2}{c}{ CVMDF } & \multicolumn{2}{c}{ FC } & \multicolumn{2}{c}{ IC } & \multicolumn{2}{c}{ FH } \\
\cline { 2 - 11 } & GL $^{2}$ & $\mathrm{~F}^{3}$ & Sig. & F & Sig. & F & Sig. & F & Sig. & F & Sig. \\
\hline Fase & 1 & 0.032 & $*$ & 0.000 & $* * *$ & 0.001 & $* * *$ & 0.000 & $* * *$ & 0.000 & $* * *$ \\
Sexo & 1 & 0.740 & n.s. & 0.537 & n.s. & 0.751 & n.s. & 0.242 & n.s. & 0.242 & n.s. \\
Clase & 1 & 0.001 & $* * *$ & 0.135 & n.s. & 0.003 & $* *$ & 0.180 & n.s. & 0.180 & n.s. \\
Fase:Sexo & 1 & 0.722 & n.s. & 0.587 & n.s. & 0.765 & n.s. & 0.350 & n.s. & 0.350 & n.s. \\
Fase:Clase & 1 & 0.812 & n.s. & 0.382 & n.s. & 0.899 & n.s. & 0.945 & n.s. & 0.945 & n.s. \\
Sexo:Clase & 1 & 0.024 & $*$ & 0.089 & n.s. & 0.076 & n.s. & 0.168 & n.s. & 0.168 & n.s. \\
Fase:Sexo: & 1 & 0.944 & n.s. & 0.816 & n.s. & 0.890 & n.s. & 0.848 & n.s. & 0.848 & n.s. \\
Clase & & & & & & & & & & &
\end{tabular}

${ }^{1}$ MDF; diámetro medio de fibra; CVMDF: coeficiente de variación de MDF; FC: factor de confort; IC: índice de curvatura; FH: finura al hilado

${ }^{2}$ Grados de libertad; ${ }^{3}$ Valor de F; ${ }^{4}$ Nivel de significancia estadística

considerado en la confección de prendas de vestir (McGregor, 2012). No obstante, la baja eficiencia del descerdado manual, convendría la implementación del descerdado mecánico y una selección genética (Frank, 2011). Esto permitiría una mayor cantidad de fibra fina en menor tiempo y una mayor generación de recursos económicos a los productores y trabajadores artesanales ligados a la producción llamera (Quispe et al., 2015).

La clase o grupo etario tuvo un efecto significativo sobre MDF y FC. Esto ha sido demostrado en llamas (Sunari, 1986; Cancino et al., 2006; Rodríguez, 2007; Frank, 2011; Quispe, 2014), en alpacas (Wang et al., 2003; McGregor y Butler, 2004; Lupton et al., 2006; Quispe et al., 2007, 2010) y en vicuñas (Quispe et al., 2010), indicando que la fibra se hace más gruesa a medida que incrementa la edad del animal.

El sexo no mostró un efecto significativo sobre las características tecnológicas estudiadas, coincidiendo con otros reportes en llamas (Martínez et al., 1997; Siguayro, 2009), alpacas (MgGregor y Butler, 2004; MgGregor, 2006; Lupton et al., 2006) y vicuñas (Quispe et al., 2007, 2010). No obstante, Mamani et al. (2012) reportaron que el sexo tendría efecto en MDF, pero esto pudo deberse al reducido tamaño de muestra que consideraron (40 llamas).

\section{Efecto de la Clase y el Sexo Según la Fase}

La clase y el sexo muestran el mismo efecto sobre las cinco características en estudio, tanto antes como después del descerdado (Cuadro 3), con excepción del CVMDF, donde la clase o grupo etario no presentó un efecto significativo en las fibras sin descerdar. No obstante la ausencia de significancia estadística entre juveniles y adultos, es posible que este factor sea mayor en adultos dado que tienen mayor cantidad de fibras gruesas («cerdas») que los animales jóvenes (Rodríguez, 2007).

Los resultados corroboran las conclusiones de diversos investigadores que han trabajado con fibras de camélidos sudamerica- 
Cuadro 3. Promedio \pm error estándar del efecto de clase (fibra sin descerdar y descerdada) y sexo sobre las características tecnológic as de la fibra de llama Chaku antes y después del descerdado

\begin{tabular}{|c|c|c|c|c|c|c|}
\hline & $\mathrm{N} .^{\circ}$ & $\begin{array}{l}\text { MDF } \\
(\mu \mathrm{m})\end{array}$ & $\begin{array}{c}\text { CVMDF } \\
\%\end{array}$ & $\begin{array}{c}\mathrm{FC} \\
\%\end{array}$ & $\begin{array}{c}\mathrm{IC} \\
(\% / \mathrm{mm})\end{array}$ & $\begin{array}{l}\mathrm{FH} \\
(\mu \mathrm{m})\end{array}$ \\
\hline \multicolumn{7}{|c|}{ Fibra sin descerdar } \\
\hline Clase & & * & n.s. & $*$ & n.s. & $*$ \\
\hline Juveniles & 113 & $21.9^{\mathrm{b}} \pm 0.3$ & $22.2^{\mathrm{a}} \pm 0.3$ & $93.3^{\mathrm{a}} \pm 2.1$ & $37.2^{\mathrm{a}} \pm 0.8$ & $21.6^{\mathrm{a}} \pm 0.3$ \\
\hline Adultos & 114 & $23.1^{\mathrm{a}} \pm 0.4$ & $22.1^{\mathrm{a}} \pm 0.3$ & $90.5^{\mathrm{b}} \pm 2.4$ & $38.3^{\mathrm{a}} \pm 0.7$ & $22.7^{\mathrm{b}} \pm 0.4$ \\
\hline Sexo & & n.s. & n.s. & n.s. & n.s. & n.s. \\
\hline Macho & 112 & $22.5^{\mathrm{a}} \pm 0.3$ & $22.3^{\mathrm{a}} \pm 0.3$ & $92.0^{\mathrm{a}} \pm 2.4$ & $37.7^{\mathrm{a}} \pm 0.8$ & $22.2^{\mathrm{a}} \pm 0.4$ \\
\hline Hembra & 115 & $22.5^{\mathrm{a}} \pm 0.4$ & $22.0^{\mathrm{a}} \pm 0.2$ & $91.9^{\mathrm{a}} \pm 2.2$ & $37.9^{\mathrm{a}} \pm 0.8$ & $22.1^{\mathrm{a}} \pm 0.4$ \\
\hline \multicolumn{7}{|c|}{ Fibra descerdada } \\
\hline Clase & & $*$ & $*$ & $*$ & n.s. & $*$ \\
\hline Juveniles & 113 & $21.3^{\mathrm{b}} \pm 0.3$ & $20.6^{\mathrm{a}} \pm 0.2$ & $95.6^{\mathrm{a}} \pm 2.1$ & $41.8^{\mathrm{a}} \pm 0.9$ & $20.7^{\mathrm{a}} \pm 0.3$ \\
\hline Adultos & 114 & $22.3^{\mathrm{a}} \pm 0.3$ & $20.1^{\mathrm{b}} \pm 0.2$ & $93.5^{\mathrm{b}} \pm 2.3$ & $43.0^{\mathrm{a}} \pm 0.8$ & $21.5^{\mathrm{b}} \pm 0.3$ \\
\hline Sexo & & n.s. & n.s. & n.s. & n.s. & n.s. \\
\hline Macho & 112 & $21.7^{\mathrm{a}} \pm 0.3$ & $20.4^{\mathrm{a}} \pm 0.2$ & $94.8^{\mathrm{a}} \pm 2.3$ & $41.6^{\mathrm{a}} \pm 0.8$ & $21.0^{\mathrm{a}} \pm 0.3$ \\
\hline Hembra & 115 & $21.9^{\mathrm{a}} \pm 0.3$ & $20.3^{\mathrm{a}} \pm 0.2$ & $94.4^{\mathrm{a}} \pm 2.2$ & $43.3^{\mathrm{a}} \pm 0.8$ & $21.2^{\mathrm{a}} \pm 0.3$ \\
\hline
\end{tabular}

nos, quienes indican que la MDF y la FH aumentan y el FC disminuye progresivamente a medida que aumenta la edad del animal (Sunari, 1986; Wang et al., 2003; McGregor y Butler, 2004; Lupton et al., 2006; Cancino et al., 2006; Rodríguez, 2007; Quispe et al., 2007, 2010; Frank, 2011). También se corroboraría que el sexo no tiene efecto sobre estas características (Martinez et al., 1997; Siguayro, 2009), sea antes o después del descerdado. Esto mismo se observa en fibras de otros camélidos sudamericanos (MgGregor y Butler, 2004; MgGregor, 2006; Lupton et al., 2006; Quispe et al., 2010).

Teniendo en cuenta estos hallazgos, en los trabajos de mejoramiento genético de llamas se debería considerar el factor clase (o edad), más no el sexo, como factor dentro de los modelos genéticos a utilizar.

\section{Conclusiones}

El descerdado y la edad de la llama tienen efectos significativos sobre la finura y su variación, el factor de confort, el índice de curvatura y la finura al hilado, mientras que el sexo no muestra ningún efecto sobre estas características.

\section{Literatura Citada}

1. Adot OG, de Cossio AP, Maguire A, 2008. Industrialization and commercialisation of vicuña, guanaco and llama fibres. En: Frank E, Antonini M, Toro O (eds). South American Camelids Research. Vol 2. Germany: Wageningen Academic Publishers. p 359-366. 
2. Cancino A, Rebuffi G, Muller JP, Duga L, Rigalt F. 2006. Parámetros cualicuantitativos de la producción de fibra de llamas (Lama glama) machos en la puna argentina. En: IV Congreso Mundial de Camélidos. Catamarca, Argentina.

3. Coates W, Ayerza R. 2004. Comparison of llama fiber obtained from two production regions of Argentina. J Arid Environs 58: 513-524. doi:10.1016/ j.jaridenv.2003.11.003

4. Cochi N. 1999. Determinación del rendimiento y calidad de la fibra descerdada de llamas (Lama glama). Tesis de Ingeniero Agrónomo. La Paz: Univ Mayor de San Andrés. 120 p.

5. Frank EN, Hick MV, Adot OG. 2011. Descriptive differential attributes of type of fleeces in llama fiber and its textile consequence. Part 2: consequences of the dehairing. J Text I 102: 41-49. doi: 10.1080/00405000903474873

6. Frank EN, Hick MV, Adot OG 2012. Determination of dehairing, carding, combing and spinning difference from lama type of fleeces. Internat J Appl Sci Technol 2: 61-70.

7. Frank EN. 2011. Producción de fibra en camélidos sudamericanos. Avances en su procesamiento y mejoramiento genético. Arch Latinoam Prod Anim 19: 16-19.

8. Hick MVH, Lamas HE, Echenique J, Prieto A, Castillo MF, Frank EN. 2009. Estudio demográfico de los atributos morfológicos y productivos en poblaciones de llamas (Lama glama) de la provincia de Jujuy, Argentina. Anim Genet Resour Informat 45: 71-78 doi: 10.1017/S1014233909990368

9. [INEI] Instituto Nacional de Estadística e Informática. 2012. Base de datos del IV Censo Nacional Agropecuario. [Internet]. Disponible en: http:// censos.inei.gob.pe/cenagro/tabulados/

10. Lupton C, McColl A, Stobart R. 2006. Fiber characteristics of the Huacaya alpaca. Small Ruminant Res 64:211-224. doi: 10.1016/j.smallrumres.2005.04.023

11. Mamani, W, Calsín, B, Quispe J. 2012. Diámetro de fibra y pelos de llamas K'ara y Ch'acu del CIP La Raya, UNA - Puno. Rev ALLPAK'A 16: 51-57.

12. Martinez M. 2009. $R$ for biologist. Tennessee, USA. [Internet]. Disponible en: https://cran.r-project.org/doc/contrib/ Martinez-RforBiologistv1.1.pdf

13. Martínez Z, Iñiguez LC, Rodríguez T. 1997. Influence of effects on quality traits and relationships between traits of the llama fleece. Small Ruminant Res 24: 203-212. doi: 10.1016/S0921-4488(96) 00925-X

14. McGregor B, Butler K. 2004. Sources of variation in fiber diameter attributes of Australian alpacas and implications for fleece evaluation and animal selection. Aust J Agr Res 55: 433-442. doi:10.1071/AR03073

15. McGregor B. 2002. Comparative productivity and grazing behaviour of Huacaya alpacas and Peppin Merino sheep grazed on annual pastures. Small Ruminant Res 44: 219-232. doi: 10.1016/ S0921-4488(02)00050-0

16. McGregor B. 2006. Production attributes and relative value of alpaca fleeces in southern Australia and implications for industry development. Small Ruminant Res 61: 93-111. doi: 10.1016/j.smallrumres.2005.07.001

17. McGregor B. 2012. Properties, processing and performance of rare natural animal fibres: a review and interpretation of existing research results. Publication 11/150. Geelong, Australia: Ed Rural Industries Research and Development Corporation. $118 \mathrm{p}$.

18. Montes M, Quicaño I, Quispe E, Quispe L, Alfonso L. 2008. Quality characteristics of Huacaya alpaca fiber produced in the Peruvian andean plateau region of Huancavelica. Span J Agric Res 6(1): 33-38. doi: 10.5424/sjar/20080615258 
19. Mueller JP, Rigalt F, Cancino AK, Lamas H. 2010. Calidad de las fibras de camélidos sudamericanos en Argentina. En: International Simposium on Fiber South American Camelids. Huancavelica, Perú.

20. Pilco SR, Delgado J, Ayala C. 2013. Efecto del descerdado manual sobre la calidad de fibra de llamas. Madrid: Ed Academia Española. 100 p.

21. Pinares R, Chipa L, Paúcar R., Quispe EC. 2014. Estudio de la diferencia post y pre descerdado de cinco características textiles de la fibra de llama (Lama glama) Ch'aku. Rev Investig Sci Sociales Tec 1: 69-77.

22. Quispe EC. 2010. Evaluación de características productivas y textiles de la fibra de alpacas Huacaya de la región de Huancavelica, Perú. En: International Simposium on Fiber South American Camelids. Huancavelica, Perú.

23. Quispe JL. 2014. Caracterización fenotípica de llamas del tipo T'amphulli conservadas en condición in situ en las regiones de Quetena Grande - Potosí y Calientes - Cochabamba. Tesis de Magíster. Cochabamba: Univ Mayor de San Simón. 105 p.

24. Quispe EC, Chipa L, Pinares R. 2015. Análisis económico y de la producción del descerdado manual de la fibra de llamas (Lama glama) Chaku. Archiv Zootec 64: 191-198.

25. Quispe EC, Flores A, Alfonso L, Galindo A. 2007. Algunos aspectos de la fibra y peso vivo de alpacas Huacaya de color blanco en la región de Huancavelica. En: XX Reunión ALPA. Cusco: Asociación Latinoamericana de Producción Animal.

26. Quispe EC, Ramos H, Mayhua P, Alfonso L. 2010. Fibre characteristics of vicuña (Vicugna vicugna mensalis). Small Ruminant Res 93: 64-66. doi:10.1016/j.smallrumres.2010.03.019
27. Quispe EC, Rodríguez T, Iñíguez L, Mueller JP. 2009. Producción de fibra de alpaca, llama, vicuña y guanaco en Sudamérica. Anim Genet Resour Informat 45: 1-14. doi: 10.1017/S1014233 909990277

28. R Core Team. 2014. R: A language and environment for statistical computing. $\mathrm{R}$ Foundation for Statistical Computing, Vienna, Austria. [Internet]. Disponible en: http://www.R-project.org/

29. Revelle M. 2015. Procedures for psychological, psychometric, and personality research. Illinois, USA. [Internet]. Disponible en: http:// personality-project.org/r/psych

30. Rodríguez T. 2007. Producción de fibra de camélidos, calidad de fibra de llama descerdada y clasificada. En: Cardozo A (ed). Camélidos. Bolivia, Cochabamba: Centro de Investigaciones en Forrajes «La Violeta». p 361-374.

31. Siguayro R. 2009. Comparación de las características físicas de las fibras de la llama Ch'aku (Lama glama) y la alpaca Huacaya (Vicugna pacos) del Centro Experimental Quimsachata del INIAPuno. Tesis de Magíster. Lima: Univ Nacional Agraria La Molina. 88 p.

32. Stemmer A, Valle Zárate A, Nuemberg $N$, Delgado J, Wurzinger M, Soelkner J. 2005. La llama de Ayopaya: Descripción de un recurso genético autóctono. Arch Zootec 54: 253-259.

33. Sunari E. 1986. Biometría de la llama en la comunidad de Santa Rosa de Juli. Tesis de Médico Veterinario y Zootecnista. Puno: Univ Nacionaldel Altiplano. $78 \mathrm{p}$.

34. Wang X, Wang L, Liu X. 2003. The quality and processing performance of alpaca fibres. Rural Industries Research and Development Corporation. Australia. $119 \mathrm{p}$. 